\title{
Modeling the grain growth kinetics of doped nearly fully
}

\section{dense nanocrystalline ceramics}

\author{
M. M. Gong ${ }^{\mathrm{a}, \mathrm{b}}$, C. H. Chang ${ }^{\mathrm{b}}$, L. J. Wu ${ }^{\mathrm{b}}$, S. Dey ${ }^{\mathrm{b}}$, R. H. R. Castro ${ }^{\mathrm{b}^{*}}$, F. Liu ${ }^{\mathrm{a}^{*}}$ \\ ${ }^{a}$ State Key Laboratory of Solidification Processing, Northwestern Polytechnical \\ University, Xi'an, Shaanxi 710072, P.R. China \\ ${ }^{\mathrm{b}}$ Department of Materials Science \& Engineering, University of California, Davis, \\ CA 95616, USA
}

\begin{abstract}
A grain growth model to describe dopant effects on nanocrystalline ceramics is proposed by incorporating the dopant-segregation-dependent grain boundary (GB) energy and the GB mobility subjected to intrinsic drag and pore drag (both affected by dopant segregation) into the parabolic growth formula. The model addresses the common case of residual porosity in grain growth behavior. Taking near-fully dense nanocrystalline lanthanum doped Yttria stabilized Zirconia (La doped
\end{abstract} YSZ) as the system of study, the grain growth behavior was explored using the model. The substantially suppressed grain growth in La doped YSZ as compared to La-free YSZ could be attributed to the combined effect of thermodynamically reduced GB energy and kinetically reduced GB mobility. Contrary to previous assumptions, the model suggests that, relative to the GB energy overall effect, the effect of the dopant on the GB mobility plays a more significant role in reducing coarsening. Furthermore, model calculation shows that both intrinsic drag and pore drag makes certain

- The corresponding authors

- Email: liufen@nwpu.edu.cn. Tel: 0086-29-88460374. Fax : 0086-29-88491484

- Email: rhrcastro@ucdavis.edu. Tel: 1 (530) 752-3724. Fax: 1 (530) 752-9307 
contribution to the evolution of GB mobility during the grain growth.

Keywords: Grain growth; dopant; segregation; YSZ

\section{Introduction}

Nanocrystalline ceramics, due to their excellent mechanical and physical properties induced by extremely tiny grain sizes, have great potential of application in industry [1-7]. However, enlarged grain boundary (GB) area in these systems compared with their bulk counterpart renders them thermodynamically unstable against grain growth. Significant grain coarsening often occurs upon their preparation process or the service condition when exposed at moderate to high temperatures. Given this, considerable efforts have been addressed to the issue of how to retard the grain growth of ceramics [8-26]. Introduction of dopants has been demonstrated as an effective way to reduce the grain sizes of and to refine the microstructures of ceramics [12-26], which, basically, can be attributed to thermodynamically reduced GB energy and/ or kinetically reduced GB mobility.

The decrease in GB energy caused by doping is generally interpreted as a consequence of segregation of solute/dopant cations at the GBs [27-34]. Weissmuller [27] first formulized this concept based on Gibbs adsorption isotherm [28] to propose an analytical expression of segregation-dependent GB energy. After that, a number of theoretical efforts [29-34] were carried out on the topic, providing good agreement with related experimental results.

Concerning the fact that ceramics are often not fully dense (densities are typically at $95 \%$ to $98 \%$ of the theoretical density), in addition to the fact that dopants tend to 
affect differently the GB energy and surface (pore) energy, the underlying mechanism describing doping effects on the GB mobility is complicated due to the interaction between GBs and the pores situated in GBs and triple junctions [35-40]. Dopants can act to alter GB diffusivity and surface diffusivity [40] to respectively affect intrinsic drag and pore drag, thereby impacting the GB mobility. Therefore, an overall consideration of these effects is required in an attempt to provide a comprehensive understanding of the influence of dopants on GB mobility. Based on the above analysis, in this work, quantitative evaluation of the effect of dopants on GB mobility will be performed, and then a modified grain growth equation is proposed by incorporating the derived GB mobility and the segregation-dependent GB energy into the parabolic grain growth formula. The model is then applied to experimental data on grain growth of nanocrystalline zirconia and critically discussed.

\section{Model derivation}

Normal (i.e. curvature-driven) gran growth in polycrystalline materials generally follows [41]:

$$
\frac{\mathrm{d} G}{\mathrm{~d} t}=\frac{M \gamma_{g b}}{G}
$$

where $G$ is the grain size (diameter), $t$ the dwelling time, $M$ the GB mobility and $\gamma_{g b}$ the GB energy. When GB energy and GB mobility remain constant with respect to the change in grain size, an integration of Eq. (1) yields the well-known parabolic growth formula.

\subsection{Dopant-dependent GB energy}

In the case of doping, the GB energy can be reduced as a result of 
solute/dopant-cation segregation at GBs [27-34]. The model of GB energy developed by Krill is adopted here to evaluate the effect of segregation on the GB energy. It reads [31]:

$$
\gamma_{g b}=\gamma_{g b 0}+\Gamma_{g b} \Delta H_{s e g}^{g b} \approx \gamma_{g b 0}+\frac{m_{g b} x_{g b}}{a^{2} N_{A v g}} \Delta H_{s e g}^{g b}
$$

where $\gamma_{g b 0}$ is the GB energy of pure solvent (host material), $\Delta H_{s e g}{ }^{g b}$ the segregation enthalpy of GB, and $\Gamma_{g b}$ the Gibbs solute excess at GBs, which can be approximated as $m_{g b} x_{g b} / a^{2} N_{A v g}$ with $m_{g b}$ the number of layers of atoms/cations within GBs, $x_{g b}$ the solute molar fraction at GBs, $a$ the interatomic distance, and $N_{\text {Avg }}$ the Avogadro's constant. According to Eq. (2), a reduction of GB energy will be predicted at a negative segregation enthalpy, corresponding to spontaneous GB segregation.

\subsection{GB mobility influenced by intrinsic drag and pore drag}

For doped, porous materials, the GB mobility would consist of the contributions from intrinsic drag $\left(M_{\text {intr }}\right)$ and pore drag $\left(M_{\text {pore }}\right)$ :

$$
\frac{1}{M}=\frac{1}{M_{\text {intr }}}+\frac{1}{M_{\text {pore }}}
$$

Each contribution will be described in details in the following sessions.

\subsubsection{Dopant-dependent intrinsic drag}

Based on Burke-Turnbull's reaction rate theory [41], the contribution from intrinsic drag, $M_{\text {intr }}$ is proportional to the GB self-diffusion coefficient $D_{g b}$. Borisov's semi-empirical equation [42], providing a link of GB and bulk self-diffusivities to GB energy, has been previously combined with the expression of segregation-dependent GB energy to allow for a quantitative description of GB self-diffusivity affected by 
segregation [43-50], such that:

$$
\gamma_{g b}=\frac{k T}{\alpha a^{2}} m_{g b}\left[\ln \left(\frac{\delta_{g b} \theta_{g b}}{a \lambda^{\alpha}}\right)-\ln m_{g b}\right]
$$

where $k$ is Boltzmann constant, $T$ the temperature (in unit of K), $\alpha$ denoting the diffusion mechanism (2 for vacancy diffusion and 1 for interstitial diffusion), $\delta_{g b}$ the width of GBs (equal to $m_{g b} a$ ), $\theta_{g b}=D_{g b} / D_{b}$ with $D_{b}$ the bulk self-diffusion coefficient, and $\lambda$ a term relating to the frequencies and potential energies of the equilibrium and activated states of a diffusing particle, calculated by Borisov et al. [42] to have an approximated value of unity. If the change in bulk self-diffusivity with respect to dopant can be neglected ${ }^{1}$, combining Eq. (4) with Eq. (2) approximately gives,

$$
D_{g b}=D_{g b 0} \exp \left(\frac{\alpha \Delta H_{s e g}^{g b}}{R T} x_{g b}\right)
$$

with $D_{g b 0}$ being the GB self-diffusivity of pure host. Furthermore, the intrinsic GB mobility influenced by doping is derived according to its linear relationship with GB self-diffusion coefficient as,

$$
M_{\mathrm{int} r}=M_{\mathrm{int} r 0} \exp \left(\frac{\alpha \Delta H_{\text {seg }}^{g b}}{R T} x_{g b}\right)
$$

where $M_{\text {intr } 0}$ is the value for the undoped material. Clearly, a negative GB segregation enthalpy, enabling positive GB segregation, would lead to a decrease in intrinsic GB mobility.

\subsubsection{Dopant-dependent pore drag}

In the situation where pores exist and are mainly located at the GBs, the

\footnotetext{
${ }^{1}$ Introduction of dopant cations into the host lattice would change the intrinsic defect equilibrium, e.g. the equilibrium concentrations of cation and anion vacancies in ceramics, thereby altering the bulk diffusivity. However, in the case of isovalent dopant, the dopant cation would likely have a weak influence on the defect equilibrium, enabling Eq. (5) still to be applicable.
} 
contribution of pore drag to the GB mobility assuming a surface diffusion dominating pore motion $^{2}$ was previously formulated by Brook [40] as,

$$
M_{\text {pore }}=\frac{\beta D_{s} \delta_{s} V_{m}}{R T G^{2}}(1-\rho)^{-4 / 3}
$$

where $\beta$ is a scaling factor, $D_{s}$ is the surface self-diffusivity, $\delta_{s}$ the width of surface diffusion layer, $V_{m}$ the molar volume and $\rho$ the relative density. It has been shown [53] that the problem of surface diffusion is fundamentally equivalent to that of GB diffusion. Hence, analogously to Eq. (5), the dopant-segregation-dependent surface self-diffusivity may be given by:

$$
D_{s}=D_{s 0} \exp \left(\frac{\alpha \Delta H_{s e g}^{s}}{R T} x_{s}\right)
$$

where $D_{s 0}$ is the surface self-diffusivity of undoped material, $\Delta H_{\text {seg }}{ }^{s}$ the segregation enthalpy of surface, and $x_{s}$ the molar fraction of solute at pore surfaces. A dopant-segregation-dependent pore mobility, $M_{\text {pore }}$, can then be derived by incorporating Eq. (8) in Eq. (7):

$$
M_{\text {pore }}=\frac{\left(\beta D_{s 0}\right) \delta_{s} V_{m}}{R T G^{2}} \exp \left(\frac{\alpha \Delta H_{\text {seg }}^{s}}{R T} x_{s}\right)(1-\rho)^{-4 / 3}
$$

The sum of the described drag forces shall lead to an effective GB mobility influenced by solute segregation following:

\footnotetext{
${ }^{2}$ Pore migration generally occurs by evaporation-condensation, surface diffusion or volume diffusion [35]. On discussions [51] on the relative importance of those various transport mechanisms, it has been shown that surface diffusion dominates for small pore radius, therefore would probably play a dominant role when the grain size is reduced to nanoscale (generally with nanoscaled pore size). Further theoretical analysis based on the experimental results has shown that pore drag controlled by surface diffusion can well account for the grain growth of nanocrystalline/ultrafine-grained ceramics, e.g. $\mathrm{TiO}_{2}, 3 \mathrm{Y}-\mathrm{TZP}, \mathrm{Y}_{2} \mathrm{O}_{3}, \mathrm{CeO}_{2}$ and $\mathrm{ZrO}_{2}$ [52], suggesting a surface-diffusion mediated pore migration.
} 


$$
M=\left[\frac{1}{M_{\text {int } r 0}} \exp \left(-\frac{\alpha \Delta H_{s e g}^{g b}}{R T} x_{g b}\right)+\frac{R T G^{2}}{\left(\beta D_{s 0}\right) \delta_{s} V_{m}}(1-\rho)^{4 / 3} \exp \left(-\frac{\alpha \Delta H_{s e g}^{s}}{R T} x_{s}\right)\right]^{-1}
$$

Furthermore, incorporating Eq. (2) and (10) into Eq. (1) gives rise to the grain growth equation for doped, porous materials:

$$
\begin{aligned}
& \frac{\mathrm{d} G}{\mathrm{~d} t}=\frac{\gamma_{g b 0}+\frac{m_{g b} x_{g b}}{a^{2} N_{A v g}} \Delta H_{s e g}^{g b}}{G} \\
& \times\left[\frac{1}{M_{\text {int } r 0}} \exp \left(-\frac{\alpha \Delta H_{s e g}^{g b}}{R T} x_{g b}\right)+\frac{R T G^{2}}{\left(\beta D_{s 0}\right) \delta_{s} V_{m}}(1-\rho)^{4 / 3} \exp \left(-\frac{\alpha \Delta H_{s e g}^{s}}{R T} x_{s}\right)\right]^{-1}
\end{aligned}
$$

Note from the equation that the grain growth rate, $\mathrm{d} G / \mathrm{d} t$, is affected by the grain size

$G$, the relative density $\rho$, and solute molar fractions at GB $\left(x_{g b}\right)$ and pore surface $\left(x_{s}\right)$. Therefore, to allow a quantitative description of grain growth, the evolution of relative density with the proceeding grain growth, as well as the effect of solute re-distribution among bulk, GB, and pore surface during growth are required.

\subsection{Evolution of relative density during grain growth}

Residual porosities found in the final stage of sintering of materials are commonly slowly eliminated concomitantly to grain growth. For nanocrystalline materials, GB diffusion can be safely assumed as the dominating densification mechanism as a result of high volume fraction of GBs. The densification rate via GB diffusion at the final stage of sintering can be described by [54]:

$$
\frac{\mathrm{d} \rho}{\mathrm{d} t}=\frac{\zeta D_{g b} \delta_{g b} \gamma_{s} V_{m}}{R T G^{4}}
$$

where $\zeta$ is also a scaling factor, and $\gamma_{s}$ is the surface energy of pores, which, in the case of surface segregation, can be described by [22, 23]: 


$$
\gamma_{s}=\gamma_{s 0}+\Gamma_{s} \Delta H_{s e g}^{s} \approx \gamma_{s 0}+\frac{m_{s} x_{s}}{a^{2} N_{A v g}} \Delta H_{s e g}^{s}
$$

with $\gamma_{s 0}$ the surface energy of the undoped material and $m_{s}$ the number of layers of atoms/cations within pore surface. Thus, by incorporating Eq. (segregation-dependent GB self-diffusion coefficient) and (13) into Eq. (12), one has:

$$
\frac{\mathrm{d} \rho}{\mathrm{d} t}=\frac{\left(\zeta D_{g b 0}\right) \delta_{g b} V_{m}}{R T G^{4}}\left(\gamma_{s 0}+\frac{m_{s} x_{s}}{a^{2} N_{A v g}} \Delta H_{s e g}^{s}\right) \exp \left(\frac{\alpha \Delta H_{s e g}^{g b}}{R T} x_{g b}\right)
$$

The equation implies that the rate of densification is dependent on the solute molar fraction at pore surface, $x_{s}$ and the grain size, $G$.

\subsection{Description of dopant re-distribution during grain growth}

The solute distribution in a polycrystalline system, including the possibility of segregation to GBs and pore surfaces [55], has been demonstrated to follow the same form as Langmuir-Mclean segregation isotherm [56]:

$$
\begin{aligned}
& \frac{x_{g b}}{1-x_{g b}}=\frac{x_{b}}{1-x_{b}} \exp \left(-\frac{\Delta H_{\text {seg }}^{g b}}{R T}\right) \\
& \frac{x_{s}}{1-x_{s}}=\frac{x_{b}}{1-x_{b}} \exp \left(-\frac{\Delta H_{s e g}^{s}}{R T}\right)
\end{aligned}
$$

Recalling mass conservation requires:

$$
\left(1-f_{g b}-f_{s}\right) x_{b}+f_{g b} x_{g b}+f_{s} x_{s}=x_{0}
$$

where $x_{0}$ is the total solute molar fraction, and $f_{g b}$ and $f_{s}$ are the site fractions occupied by GBs and pore surface, respectively. Under the approximation of a tetrakaidecahedron for grain shape [57], these can be expressed as a function of grain size and relative density [55]: 


$$
\begin{gathered}
f_{g b}==\frac{(3+6 \sqrt{3})}{8 \sqrt{2} \sqrt[3]{\frac{\pi}{48 \sqrt{2}}} \frac{\delta_{g b}}{G}} \\
f_{s}=144\left(\frac{1-\rho}{48}\right)^{\frac{2}{3}} \frac{\delta_{s}}{G}
\end{gathered}
$$

Thus, by combing Eq. (15) with Eq. (16) and (17), the solute molar fraction at GB $\left(x_{g b}\right)$ and pore surface $\left(x_{s}\right)$ can be related to grain size and relative density. Therefore, the evolution of grain size as a function of annealing time is obtained by combining Eq. (11) (the grain growth equation) with Eq. (14) (the densification equation) and Eq. (15-17) (segregation equations).

\section{Model application and discussion}

Yttria-stabilized zirconia (YSZ) has a wide range of applications in industry due to its excellent physical and mechanical properties [58-60] and was therefore chosen as the host system for the model application. Moreover, recently, the dopant $\mathrm{La}^{3+}$, with identical charge and oversized ionic radius as compared with $\mathrm{Y}^{3+}$, has been demonstrated to slow down grain growth in this system [24]. This was attributed to significant segregation (driven by radii differences) to interfaces, with a respective minimal effect on the defect equilibrium and thus on the bulk diffusion (due to the identical charges), making it a suitable model system as it matches the assumptions of the proposed model, as described in section 2.2.1.

\subsection{Description of the grain growth of nanocrystalline 12YSZ and 2La10YSZ}

$$
\text { Isothermal grain growth experiment for nanocrystalline }
$$

$12 \mathrm{~mol} \% \mathrm{Y}_{2} \mathrm{O}_{3}-88 \mathrm{~mol} \% \mathrm{ZrO}_{2}(12 \mathrm{YSZ})$ and $2 \mathrm{~mol} \% \mathrm{La}_{2} \mathrm{O}_{3}-10 \mathrm{~mol} \% \mathrm{Y}_{2} \mathrm{O}_{3}-88 \mathrm{~mol}^{2} \mathrm{ZrO}_{2}$

(2La10YSZ) were performed in our previous study [24]. The pellets were placed on a 
platinum coated alumina boat in a horizontal tube furnace (Lindberg/blue. M. Inc.), and heated up to $1100{ }^{\circ} \mathrm{C}$ for the appropriate dwell time. Grain sizes after grain growth experiment were measured by either X-ray diffraction (XRD; model D8 Advance, Bruker AXS Inc., Madison, WI) using whole profile fitting refinement or scanning electron microscopy (SEM; FEI 430 Nano-SEM instrument, FEI Company, Hillsboro, Oregon).

As shown in Fig. 1, significant grain growth occurred for both 12YSZ and 2La10YSZ while 2La10YSZ presented a strong retardation in grain growth as compared to 2La10YSZ. The fluorite structure was always kept during grain growth of both 12YSZ and 2La10YSZ, without any precipitation of solute-rich phases even after an isothermal annealing of $48 \mathrm{~h}$, see Ref. [24]. Scanning Electron Microscopy (SEM) images of $12 \mathrm{YSZ}$ and $2 \mathrm{La10YSZ}$ at different annealing stages are shown in Fig. 2. Along with the grain coarsening, the porosity of both 12YSZ and 2La10YSZ decreased with prolonging the annealing time. By means of Archimedes' method, the relative density was measured, increasing from $\sim 94 \%(\sim 92 \%)$ in the beginning to $\sim 97 \%$ $(\sim 98 \%)$ in the end of annealing for nanocrystalline 12YSZ (2La10YSZ). It should be noted that most pores were situated in the GBs before grain growth studyies started, which is well in line with the model assumptions cited in section 2.2.2.

The next session addresses the procedures utilized to check the applicability of the model to elucidate mechanisms behind grain growth of 12YSZ and 2La10YSZ.

\subsection{Procedure of model application and determination of parameters}

For 12YSZ, the GB energy was assumed to remain isotropic and constant with 
respect to the change of grain size and relative density. Hence, the GB mobility comprising the contributions from intrinsic drag and pore drag can be described by:

$$
M=\left[\frac{1}{M_{\mathrm{int} r 0}}+\frac{R T G^{2}}{\left(\beta D_{s 0}\right) \delta_{s} V_{m}}(1-\rho)^{4 / 3}\right]^{-1}
$$

The grain growth equation in this case is then derived by incorporating Eq. (18) into Eq. (1):

$$
\frac{\mathrm{d} G}{\mathrm{~d} t}=\left[\frac{1}{M_{\text {int } r 0}}+\frac{R T G^{2}}{\left(\beta D_{s 0}\right) \delta_{s} V_{m}}(1-\rho)^{4 / 3}\right]^{-1} \frac{\gamma_{g b 0}}{G}
$$

Accordingly, both the GB self-diffusion coefficient and the surface energy are assumed invariable so that the densification equation is rewritten as:

$$
\frac{\mathrm{d} \rho}{\mathrm{d} t}=\frac{\left(\zeta D_{g b 0}\right) \delta_{g b} \gamma_{s 0} V_{m}}{R T G^{4}}
$$

A combination of Eq. (19) with Eq. (20) will then give the evolution of grain size as a function of time for nanocrystalline 12 YSZ.

$\mathrm{La}^{3+}$ has been demonstrated to segregate strongly to GBs and pore surface in La doped YSZ $[24,55]$. Therefore, a considerable influence on GB/surface energy and $\mathrm{GB} /$ surface self-diffusion is expected, thereby affecting the microstructural evolution of nanocrystalline 2La10YSZ. A description of the grain growth behavior for nanocrystalline 2La10YSZ requires a combination of Eq. (11) (segregation-dependent gain growth equation) with Eq. (14) (segregation-dependent densification equation) and Eq. (15-17) (segregation equations).

The values of parameters used for the model fittings and calculations are gathered in Table 1 . Therein, a value of $1.15 \mathrm{Jm}^{-2}$ for the GB energy $\left(\gamma_{g b 0}\right)$ was obtained by the measurement of heat release upon the grain growth of 12YSZ using Differential 
Scanning Calorimetry (DSC), as detailed elsewhere [24]. The surface energy $\left(\gamma_{s 0}\right)$ for 12YSZ was determined as $1.17 \mathrm{~J} \mathrm{~m}^{-2}$ using water adsorption microcalorimetry, see ref. [55]. The segregation enthalpy of GB and surface, $\Delta H_{s e g}{ }^{g b}$ and $\Delta H_{\text {seg }}{ }^{s}$ for La in YSZ was derived to be -48.75 and $-54.80 \mathrm{~kJ} \mathrm{~mol}^{-1}$, respectively [55]. The interatomic distance $a$ was estimated from the cubic root of a unit cell volume, i.e. $\left(a_{0}^{3} / 4\right)^{1 / 3}$ with $a_{0}$ the lattice constant and the factor 4 denoting a unit cell of fluorite structure shared by 4 cations. The lattice constant of as-sintered nanocrystalline 12YSZ was adopted here, because the lattice constant calculated from the XRD patterns exhibit a weak dependence on the composition or grain size [24]. The parameter $\alpha$ is set to be 2 since cations that serve as the rate-determining species in YSZ diffuse via vacancy mechanism ${ }^{3}$. The number of atomic layers is typically assumed as 3 for GBs $\left(m_{g b}\right)$ and 1 for surface $\left(m_{s}\right)$ [22]. The parameters $\beta D_{s 0}, \zeta D_{g b 0}$, and $M_{\text {intr } 0}$ were all taken as the fitting parameters of this model.

\subsection{Comparison of experimental measurement and model calculation for grain growth}

The fittings of this model into the experimental data of grain growth for 12YSZ and 2La10YSZ were performed simultaneously with the model parameters depicted in Table 1. As shown in Fig. 1, this model represents a good description for the evolution of grain size as a function of annealing time for both 12YSZ and 2La10YSZ. The parameters, $\beta D_{s 0}, \zeta D_{g b 0}$, and $M_{\text {intr } 0}$ were fitted as $1.0 \times 10^{-17} \mathrm{~m}^{2} \mathrm{~s}^{-1}, 4.5 \times 10^{-18} \mathrm{~m}^{2} \mathrm{~s}^{-1}$, and

\footnotetext{
${ }^{3}$ The dominant mechanism for cation transport in fluorite and fluorite-like structures should be the so-called "interstitial" diffusion [25, 52-55], which, however, is clearly different from typical interstitial diffusion of moving atoms from one interstitial site to another but has the same path of atom movement as typical vacancy diffusion.
} 
$1.5 \times 10^{-18} \mathrm{~m}^{4} \mathrm{~J}^{-1} \mathrm{~s}^{-1}$, respectively. It's difficult to judge whether or not the values of these fitting parameters are reasonable since a comparison with their reference values is not available. In an attempt to check the reliability of these fitted values, the densities after $48 \mathrm{~h}$ for both $12 \mathrm{YSZ}$ and 2La10YSZ were measured, providing a comparison with those from model prediction. As described in section 3.1, the 12YSZ grains achieved $\sim 97 \%$ while the 2La10YSZ leveled at $~ 98 \%$ after this annealing time, which should be comparable, respectively, with $\sim 98 \%$ for $12 \mathrm{YSZ}$ and $\sim 100 \%$ for 2La10YSZ evaluated by this model adopting those fitting parameters. The agreement shows the feasibility of the model and the rationality of the parameters to a certain extent.

\subsection{Thermodynamic and kinetic effects of dopant segregation on grain growth}

The retarded grain growth in $12 \mathrm{YSZ}$ could be attributed to the reduction of GB mobility as a result of pore drag. The GB mobility for 12 YSZ upon this process was evaluated, decreasing from $4.9 \times 10^{-19}$ to $3.8 \times 10^{-20} \mathrm{~m}^{4} \mathrm{~J}^{-1} \mathrm{~s}^{-1}$ as the grain growth proceeds till the end of the growth. The observed mobility is much lower than the intrinsic GB mobility $\left(M_{\text {intr } 0}=1.5 \times 10^{-18} \mathrm{~m}^{4} \mathrm{~J}^{-1} \mathrm{~s}^{-1}\right)$. The contribution of pore drag to GB mobility $M_{\text {pore }}$ is inversely proportional to the grain size squared $\left(G^{2}\right)$, since this effects the area for porosity distribution, but is directly proportional to $(1-\rho)^{-4 / 3}$ as described by Eq. (7), where the density increases with grain size. As a result, the evolution of $M_{\text {pore }}$ should then hinge on the combined effect of changes of grain size and relative density. In the present case for $12 \mathrm{YSZ}$, the resultant decrease in $M_{\text {pore }}$ shows that the grain coarsening plays a more significant role in affecting the GB 
mobility as compared to the porosity change.

For 2La10YSZ, $\mathrm{La}^{3+}$ segregation at both the GBs and the free surfaces, as elucidated in section 3.2, would affect the GB energy and the GB mobility, thereby impacting on grain growth. Besides, pore drag also contributes to decrease the GB mobility. The following relation was proposed here to clarify the dominant role of the thermodynamically reduced GB energy (by segregation) or the kinetically reduced GB mobility (by both segregation and pore drag):

$$
\frac{\left.\frac{\mathrm{d} G}{\mathrm{~d} t}\right|_{\text {state } 1}}{\left.\frac{\mathrm{d} G}{\mathrm{~d} t}\right|_{\text {state } 2}}=\frac{\frac{M \gamma_{g b}}{G}}{\frac{M_{\mathrm{int} r 0} \gamma_{g b 0}}{G}}=\left(\frac{M}{M_{\mathrm{int} r 0}}\right)\left(\frac{\gamma_{g b}}{\gamma_{g b 0}}\right)
$$

where the subscript "statel" denotes the case subjected to both segregation and pore drag while the subscript "state2" represents the case where the GB energy and the GB mobility hold constant with grain growth, being their intrinsic values. The relative retardation of grain growth in the case subjected to both segregation and pore drag can be determined as the product of normalized GB energy $\left(\gamma_{g b} / \gamma_{g b 0}\right)$ and normalized GB mobility $\left(M / M_{\text {intr } 0}\right)$. One can see that if $\gamma_{g b} / \gamma_{g b 0}<M / M_{\text {intr } 0}$, the retardation of reduced GB energy on the grain growth is stronger than that of reduced GB mobility, which means the thermodynamic effect of segregation prevails over the grain growth process; if $M / M_{\text {intr } 0}<\gamma_{g b} / \gamma_{g b 0}$, the kinetic effect by both segregation and pore drag plays a dominant role in grain growth. In view of this, the evolution of $\gamma_{g b} / \gamma_{g b 0}$ and $M / M_{\text {intr } 0}$ as a function of annealing time was then calculated, as shown in Fig. 3. A remarkable reduction of $\gamma_{g b} / \gamma_{g b 0}$ is clearly observed, which is simply interpreted as a result of enhanced $\mathrm{La}^{3+}$ segregation to the GBs in 2LaYSZ associated with the decrease of GB 
area, whereas, $M / M_{\text {intro }}$ first decreases rapidly and then increases slightly with prolonging the annealing time, which would be attributed to the combined effect of segregation and pore drag. One can note that during the whole growth process, $M / M_{\text {intro }}$ is apparently smaller than $\gamma_{g b} / \gamma_{g b 0}$, suggesting the kinetic effect of segregation as well as pore drag is playing a dominant role in retarding the grain growth of nanocrystalline 2La10YSZ.

Fig. 4 shows the relative contribution of intrinsic drag $\left(M_{\text {intr }}\right)$ and pore drag $\left(M_{\text {pore }}\right)$ to GB mobility and the GB mobility itself $(M)$ during the grain growth process. One observes that $M_{\text {intr }}$ always decreases, whereas $M_{\text {pore }}$ first decreases and then increases as the grain growth proceeds, resulting in $M$ increasing at first and then staying nearly constant. The evolution of $M_{\text {intr }}$ results from the enhanced GB segregation of $\mathrm{La}^{3+}$ as a result of the decrease in GB area, whereas, based on Eq. (9), the factors influencing $M_{\text {pore }}$ are various, including the time-dependent relative density (porosity), grain size and surface segregation of $\mathrm{La}^{3+}$. As shown in Fig. 2, significant grain growth and observable relative-density increase (i.e. porosity decrease) for 2La10YSZ were clearly verified; according to Eq. (9), grain coarsening acts to reduce $M_{\text {pore }}$ while the increase in relative density has an opposite effect. Similarly, enhanced surface segregation of $\mathrm{La}^{3+}$ is expected as a result of the decrease in pore-surface area (corresponding to the increase of relative density). At the beginning of annealing, the decrease in $M_{\text {pore }}$ would be attributed to the combined effect of grain coarsening and enhanced surface segregation of $\mathrm{La}^{3+}$, which offsets the opposite effect of relative-density increase. After that, the raising $M_{\text {pore }}$ suggests the increase in relative 
density would play a more significant role compared to grain coarsening and enhanced surface segregation. Note that $M_{\text {pore }}$ increases very rapidly at the end of annealing time, which would be due to the relative density shortly approaching unity.

It can be inferred from Fig. 4 that the initial grain growth for 2La10YSZ would be mainly controlled by pore drag as $M_{\text {pore }}$ is far below than $M_{\text {intr }}$. With further prolonging annealing time, the increase in $M_{\text {pore }}$ and the decrease in $M_{\text {intr }}$ make them close to each other, yielding comparable pore drag and intrinsic drag. Beyond the critical point with $M_{\text {pore }}=M_{\text {intr }}$, marked by an arrow in Fig. 4 , intrinsic drag contributes more than pore drag. At the final stage of grain growth, intrinsic drag would dominate over this growth process as $M_{\text {pore }}$ is much higher than $M_{\text {intr }}$.

Based on the above analysis, it can be concluded that for 2La10YSZ both (dopant-segregation-dependent) pore drag and intrinsic drag plays a certain role in affecting the GB mobility to retard the grain growth. This effect is in addition to the thermodynamic role that seems moderate at this stage of the process, but as the grains grow, tends to zero [27, 29, 30], a condition that would overset any diffusion contribution and virtually stop growth. This could not be observed within the studied grain size range.

\section{Conclusion}

The grain growth in nanocrystalline doped ceramics in the final stage of sintering was investigated by fitting experimental data with theoretical modeling. The main conclusions are presented as follows:

1. The grain growth equation was established by incorporating the 
dopant-segregation-dependent GB energy and mobility into the parabolic growth formula of grain growth. Therein, the GB mobility, subjected to both intrinsic drag and pore drag, was obtained by a combination of the intrinsic mobility incorporating segregation-dependent GB self-diffusivity and the pore mobility incorporating segregation-dependent surface self-diffusivity.

2. Good agreements were achieved between the experimental grain growth and the model calculation for both nanocrystalline 12YSZ and 2La10YSZ. Pore drag is demonstrated to dominate over the grain growth process for nanocrystalline $12 \mathrm{YSZ}$ while the retardation of grain growth in nanocrystalline $2 \mathrm{La10YSZ}$ is attributed to the combined effect of the reduced GB energy (due to enhanced $\mathrm{La}^{3+}$ segregation at GBs) and the reduced GB mobility (due to the combined intrinsic drag and pore drag both affected by segregation).

3. For 2La10YSZ, during the initial grain growth, pore drag is found to play a major role in reducing the GB mobility. Then the role of intrinsic drag becomes comparable with that of pore drag as the grain growth proceeds. With further prolonging the annealing time to beyond a critical point with $M_{\text {intr }}=M_{\text {pore }}$, intrinsic drag has a more significant influence in GB mobility. Further coarsening is expected to meet a zero grain boundary energy condition, which is not discussed here.

\section{Acknowledgements}

The authors are grateful to the financial support of National Basic Research Program of China (No. 2011CB610403), the Natural Science Foundation of China (Nos. 51134011 and 51431008), the Fundamental Research Fund of Northwestern 
Polytechnical University (No. JC20120223), and the China National Funds for Distinguished Young Scientists (No. 51125002). M.M. Gong is thanked for the

financial supports of the Doctorate Foundation of Northwestern Polytechnical University (CX201204) and of China Scholarship Council. R. H. R. Castro is thanked for the financial support of the National Science Foundation (DMR 1609781).

\section{References}

[1] J. KARCH, R. BIRRINGER, Nanocrystalline ceramics: Possible candidates for net-shape forming, Ceram. Int. 16 (1990) 291-294.

[2] M.J. MAYO, Superplasticity of Nanostructured Ceramics, in: M. Nastasi, D.M. Parkin, H. Gleiter (Eds.), Mechanical properties and deformation behaviour of materials having ultrafine microstructures, Kluwer, Dordrecht, 1993, pp. 361-380.

[3] H. HAHN, R.S. AVERBACK, Low-Temperature Creep of Nanocrystalline Titanium (IV) Oxide, J. Am. Ceram. Soc. 74 (1991) 2918-2921.

[4] Y.S. Zhao, J. Qian, L.L. Daemen, C. Pantea, J.Z. Zhang, G.A. Voronin, T.W. Zerda, Enhancement of fracture toughness in nanostructured diamond-SiC composites, Appl. Phys. Lett. 84 (2004) 1356-1358 .

[5] S. Zhang, D. Sun, Y.Q. Fu, H.J. Du, Surf. Coat. Technol. 167 (2003) 113-119.

[6] R. W. Siegel, Nanostructures of Metals and Ceramics, in: A.S. Edelstein, R.C. Cammarata (Eds.), Nanomaterials: Synthesis, Properties and Applications, IOP, Bristol, 1996, pp. 201-218.

[7] J.A. Wollmershauser, B.N. Feigelson, E.P. Gorzkowski, C.T. Elis, R. Goswami, S.B. Qadri, J.G. Tischler, F.J. Kub, R.K. Everett, An extended hardness limit in 
bulk nanoceramics, Acta Mater. 69 (2014) 9-16.

[8] I.W. Chen, X.H. Wang, Sintering dense nanocrystalline ceramics without final-stage grain growth, Nature 404 (2000) 168-171.

[9] S. Anas, R.V. Mangalaraja, Mukundan Poothayal, Sanjeev K. Shukla, S. Ananthakumar, Direct synthesis of varistor-grade doped nanocrystalline $\mathrm{ZnO}$ and its densification through a step-sintering technique, Acta Mater. 55 (2007) $5792-5801$.

[10]M. Mazaheri, A. Simchi, F.G. Fard, Densification and grain growth of nanocrystalline 3Y-TZP during two-step sintering, J. Eur. Ceram. Soc. 28 (2008) 2933-2939.

[11]M. Mazaheri, M. Valefi, Z.R. Hesabi, S.K. Sadrnezhaad, Two-step sintering of nanocrystalline $8 \mathrm{Y}_{2} \mathrm{O}_{3}$ stabilized $\mathrm{ZrO}_{2}$ synthesized by glycine nitrate process, Ceram. Int. 35 (2009) 13-20.

[12]P.J. JORGENSEN, Modification of Sintering Kinetics by Solute Segregation in $\mathrm{Al}_{2} \mathrm{O}_{3}$, J. Am. Ceram. Soc. 48 (1965) 207-210.

[13]M.P. Harmer, R.J. Brook, The effect of $\mathrm{MgO}$ additions on the kinetics of hot pressing in $\mathrm{Al}_{2} \mathrm{O}_{3}$, J. Mater. Sci. 15 (1980) 3017-3024.

[14]K.K. Soni, A.M. Thompson, M.P. Harmer, D.B. Williams, J.M. Chabala, R.L. Setti, Appl. Phys. Lett. 66 (1995) 2795-2797.

[15]J.X. Fang, A.M. Thompson, M.P. Harmer, and H.M. Chan, Effect of Yttrium and Lanthanum on the Final-Stage Sintering Behavior of Ultrahigh-Purity Alumina, J. Am. Ceram. Soc. 80 (1997) 2005-2012. 
[16]S. Tekeli, M. Erdogan, B. Aktas, Influence of $\alpha-\mathrm{Al}_{2} \mathrm{O}_{3}$ addition on sintering and grain growth behaviour of $8 \mathrm{~mol} \% \quad \mathrm{Y}_{2} \mathrm{O}_{3}$-stabilised cubic zirconia $\left(\mathrm{c}-\mathrm{ZrO}_{2}\right)$, Ceram. Int. 30 (2004) 2203-2209.

[17]S. Tekeli, M. Erdogan, B. Aktas, Microstructural evolution in 8 mol\% $\mathrm{Y}_{2} \mathrm{O}_{3}$-stabilized cubic zirconia (8YSCZ) with $\mathrm{SiO}_{2}$ addition, Mater. Sci. Eng. A $386(2004) 1-9$.

[18]R.S. Averback, H.J. Höfler, H. Hahn, J.C. Logas, Sintering and grain growth in nanocrystalline ceramics, Nanostruct. Mater. 1 (1992) 173-178.

[19]J.G. Li, T. Ikegami, T. Mori, Low temperature processing of dense samarium-doped $\mathrm{CeO}_{2}$ ceramics: sintering and grain growth behaviors, Acta Mater. 52 (2004) 2221-2228.

[20]P. Bowen, C. Carry, From powders to sintered pieces: forming, transformations and sintering of nanostructured ceramic oxides, Powder Technol. 128 (2002) 248-255.

[21]G.S.A.M. Theunissen, A.J.A. Winnubst, A.J. Burggraaf, Sintering kinetics and microstructure development of nanoscale Y-TZP ceramics, J. Eur. Ceram. Soc. 11 (1993) 315-324.

[22]C.H. Chang, M.M. Gong, S. Dey, F. Liu, R.H.R. Castro, Thermodynamic Stability of SnO2 Nanoparticles: The Role of Interface Energies and Dopants, J. Phys. Chem. C 119 (2015) 6389-6397.

[23]L.J. Wu, J.A. Aguiar, P.P. Dholabhai, T. Holesinger, T. Aoki, B.P. Uberuaga, and R.H.R. Castro, Interface Energies of Nanocrystalline Doped Ceria: Effects of 
Manganese Segregation, J. Phys. Chem. C 119 (2015) 27855-27864.

[24]S. Dey, C.H. Chang, M.M. Gong, F. Liu, R.H.R. Castro, Grain growth resistant nanocrystalline zirconia by targeting zero grain boundary energies, J. Mater. Res. 30 (2015) 2991-3002.

[25]P.L. Chen, I.W. Chen, Role of Defect Interaction in Boundary Mobility and Cation Diffusivity of $\mathrm{CeO}_{2}$, J. Am. Ceram. Soc. 77 (1994) 2289-2297.

[26]M.N. Rahaman, R.Manalert, Grain Boundary Mobility of $\mathrm{BaTiO}_{3}$ Doped with Aliovalent Cations, J. Eur. Ceram. Soc. 18 (1998) 1063-1071.

[27]J. Weissmüller, Alloy effects in nanostructures, Nanostruct. Mater. 3 (1993) 261-272.

[28]J.W. Gibbs, Trans Conn. Acad. III (1876) 108.

[29]R. Kirchheim, Grain coarsening inhibited by solute segregation, Acta Mater. 50 (2002) 413-419.

[30]F. Liu, R. Kirchheim, Nano-scale grain growth inhibited by reducing grain boundary energy through solute segregation, J. Cryst. Growth 264 (2004) 385-391.

[31]C.E. Krill, H. Ehrhardt, R. Birringer, Thermodynamic stabilization of nanocrystallinity, Z. Metallkd. 96 (2005) 1134-1141.

[32]J.R. Trelewicz, C.A. Schuh, Grain boundary segregation and thermodynamically stable binary nanocrystalline alloys, Phys. Rev. B 79 (2009) 094112.

[33]M. Saber, H. Kotan, C.C. Koch, R.O. Scattergood, Thermodynamic stabilization of nanocrystalline binary alloys, J. Appl. Phys. 113 (2013) 063515. 
[34]M. Saber, H. Kotan, C.C. Koch, R.O. Scattergood, A predictive model for thermodynamic stability of grain size in nanocrystalline ternary alloys, J. Appl. Phys. 114 (2013) 103510.

[35]W.D. KINGERY, B. FRANCOIS, Grain Growth in Porous Compacts, J. Am. Ceram. Soc. 48 (1965) 546-547.

[36]F.A. Nichols, Theory of Grain Growth in Porous Compacts, J. Appl. Phys. 37, 4599-4602 (1966).

[37]F.A. Nichols, Further Comments on the Theory of Grain Growth in Porous Compacts, J. Am. Ceram. Soc. 51 (1968) 468-469.

[38]R.J. Brook, Pore-Grain Boundary Interactions and Grain Growth, J. Am. Ceram. Soc. 52 (1969) 56-57.

[39]R.J. Brook, Pores and Grain Growth Kinetics, J. Am. Ceram. Soc. 52 (1969) 339-340.

[40]R.J. Brook, Fabrication Principles for the Production of Ceramics with Superior Mechanical Properties, Proc. Br. Ceram. Soc. 32 (1982) 7-24.

[41]J.E. Burke, D.Turnbull, Recrystallization and grain growth, Prog. Met. Phys. 3 (1952) 220-292.

[42]V.T. Borisov, V.M. Golikov, G.V. Scherbedinskiy, Relation Between Diffusion Coefficients and Grain Boundary Energy, Fiz. Met. Metalloved, 17 (1964) $881-885$

[43]P. Nanni, C.T.H. Stoddart, E.D. Hondros, Grain boundary segregation and sintering in alumina, Mater. Chem. 1 (1976) 297-320. 
[44]D. Gupta, Influence of Solute Segregation on Grain-Boundary Energy and Self-Diffusion, Metall. Trans. A 8 (1977) 1431-1438.

[45]J. Bernardini, P. Gas, E.D. Hondros, M.P. Seah, The role of solute segregation in grain boundary diffusion, Proc. R. Soc. Lond. A 379 (1982) 159-178.

[46]E.D. Hondros, P.J. Henderson, Role of Grain Boundary Segregation in Diffusional Creep, Metall. Trans. A 14 (1983) 521-530.

[47]M. Aucouturier, Grain Boundary Segregation. Grain Boundary Diffusion, in Polycrystalline Semiconductors, Springer, Berlin, 1985.

[48]H. Riedel, Fracture at High Temperatures, Springer, Berlin, 1987.

[49]D. Gupta, Diffusion, Solute Segregations and Interfacial Energies in Some Material: An Overview, Interf. Sci. 11 (2003) 7-20.

[50]D. Prokoshkina, V.A. Esin, G. Wilde, S.V. Divinski, Grain boundary width, energy and self-diffusion in nickel: Effect of material purity, Acta Mater. 61 (2013) 5188-5197.

[51]P.G. Shewmon, The movement of small inclusions in solids by a temperature gradient, Trans. Metall. Soc. A.I.M.E. 230 (1964) 1134-1137.

[52]J. Kanters, U. Eisele, J. Rödel, Effect of initial grain size on sintering trajectories, Acta Mater. 48 (2000) 1239-1246.

[53]P. Benoist, G. Martin, Atomic model for grain boundary and surface diffusion, Thin Solid Films, 25 (1975) 181-197.

[54]S.J.L. Kang, Y. Jung, Sintering kinetics at final stage sintering: model calculation and map construction, Acta Mater. 52 (2004) 4573-4578. 
[55]M.M. Gong, S. Dey, L.J. Wu, C.H. Chang, H.Li, R.H.R. Castro, F. Liu, Effects of Concurrent Grain Boundary and Surface Segregation on the Final Stage of Sintering: the Case of Lanthanum Doped Yttria-stabilized Zirconia, J. Mater. Sci. Technol. accepted.

[56]D. McLean, Grain Boundary in Metals, Oxford University Press, London, 1957.

[57]R.L. Coble, Sintering Crystalline Solids. I. Intermediate and Final State Diffusion Models, J. Appl. Phys. 32 (1961) 787-792.

[58]N.Q. Minh, Ceramic Fuel-Cells, J. Am. Ceram. Soc. 76 (1993) 563-588.

[59]A. Selçuk, A. Atkinson, Strength and Toughness of Tape-Cast Yttria-Stabilized Zirconia, J. Am. Ceram. Soc. 83 (2000) 2029-2035.

[60]L.B. Chen, Yttria-stabilized zirconia thermal barrier coatings-a review, Surf. Rev. Lett. 13 (2006) 535-544.

[61]S.L. Hwang, I.W. Chen, Grain Size Control of Tetragonal Zirconia Polycrystals Using the Space Charge Concept, J. Am. Ceram. Soc. 73 (1990) 3269-3277.

[62]P.L. Chen, I.W. Chen, Grain Growth in $\mathrm{CeO}_{2}$ : Dopant Effects, Defect Mechanism, and Solute Drag, J. Am. Ceram. Soc. 79 (1996) 1793-1800.

[63]M. Kilo, M.A. Taylor, C. Argirusis, G. Borchardt, R.A. Jackson, O. Schulz, M. Martin, M. Weller, Modeling of cation diffusion in oxygen ion conductors using molecular dynamics, Solid State Ionics 175 (2004) 823-827.

[64] R.L.G.Romero, J.J. Meléndez, D.G.García, F.L. Cumbrera, A. D. Rodríguez, F. Wakai, Cation diffusion in yttria-zirconia by molecular dynamics, Solid State Ionics 204-205 (2011) 1-6. 


\section{Figure Captions}

Fig. 1 Evolution of grain sizes for nanocrystalline 12YSZ and 2La10YSZ as functions of annealing time at $1100 \mathrm{C}$. The solid and empty squares denote the experimental data while the solid and dashed lines represent the fitting results using the current model. 
Fig. 2 SEM photographs of nanocrystalline 12YSZ and 2La10YSZ annealed at $1100^{\circ} \mathrm{C}$ after different annealing time: (a) 12YSZ for $7 \mathrm{~h}$, (b) $12 \mathrm{YSZ}$ for $48 \mathrm{~h}$, (c) 2La10YSZ for $7 \mathrm{~h}$ and (d) 2La10YSZ for $48 \mathrm{~h}$.

Fig. 3 Normalized GB energy $\left(\gamma_{g b} / \gamma_{g b 0}\right)$ and mobility $\left(M / M_{\text {intr } 0}\right)$ calculated as functions of annealing time.

Fig. 4 Contributions from each type of drag to GB mobility, plotted as functions of annealing time. 


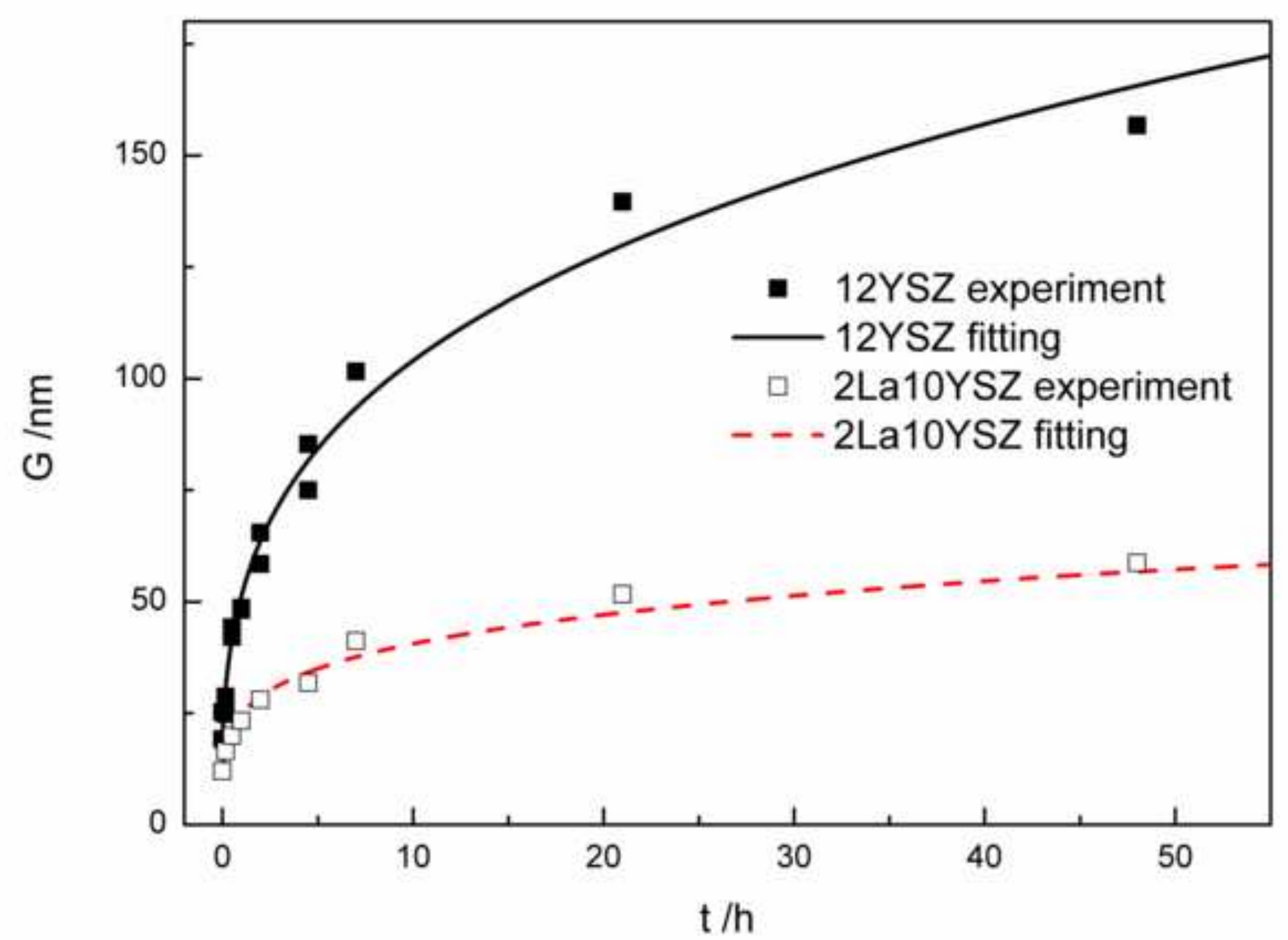




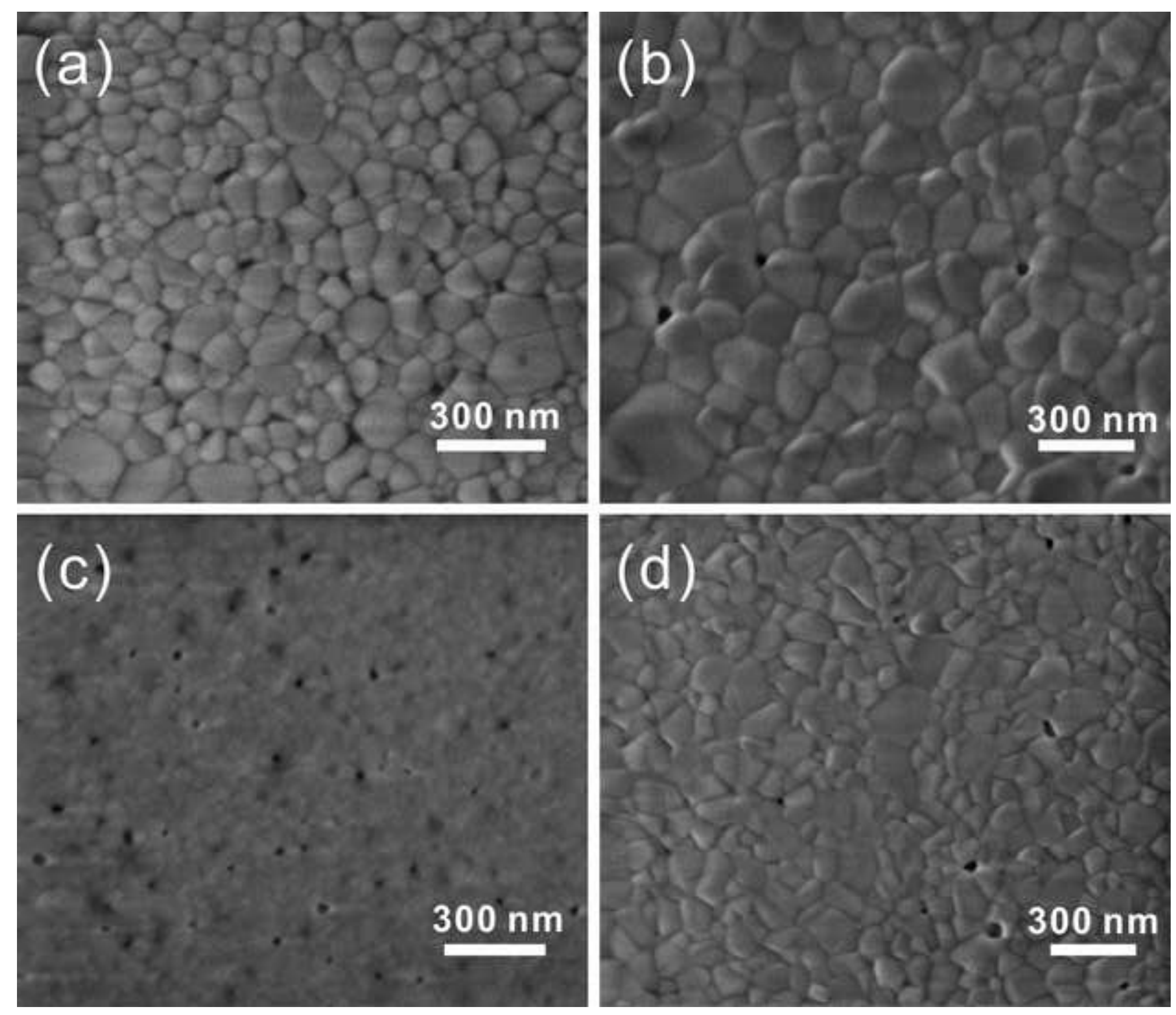




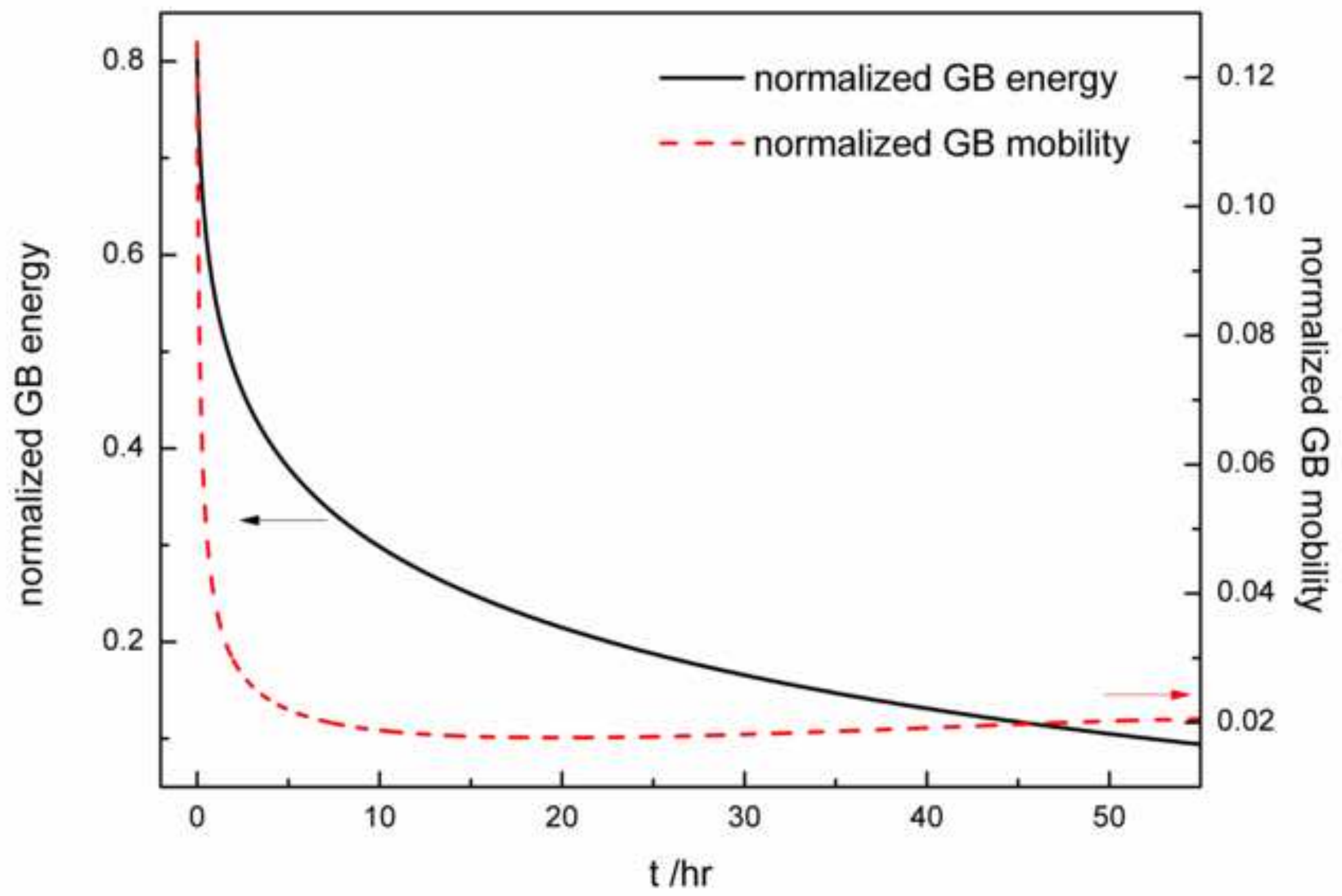




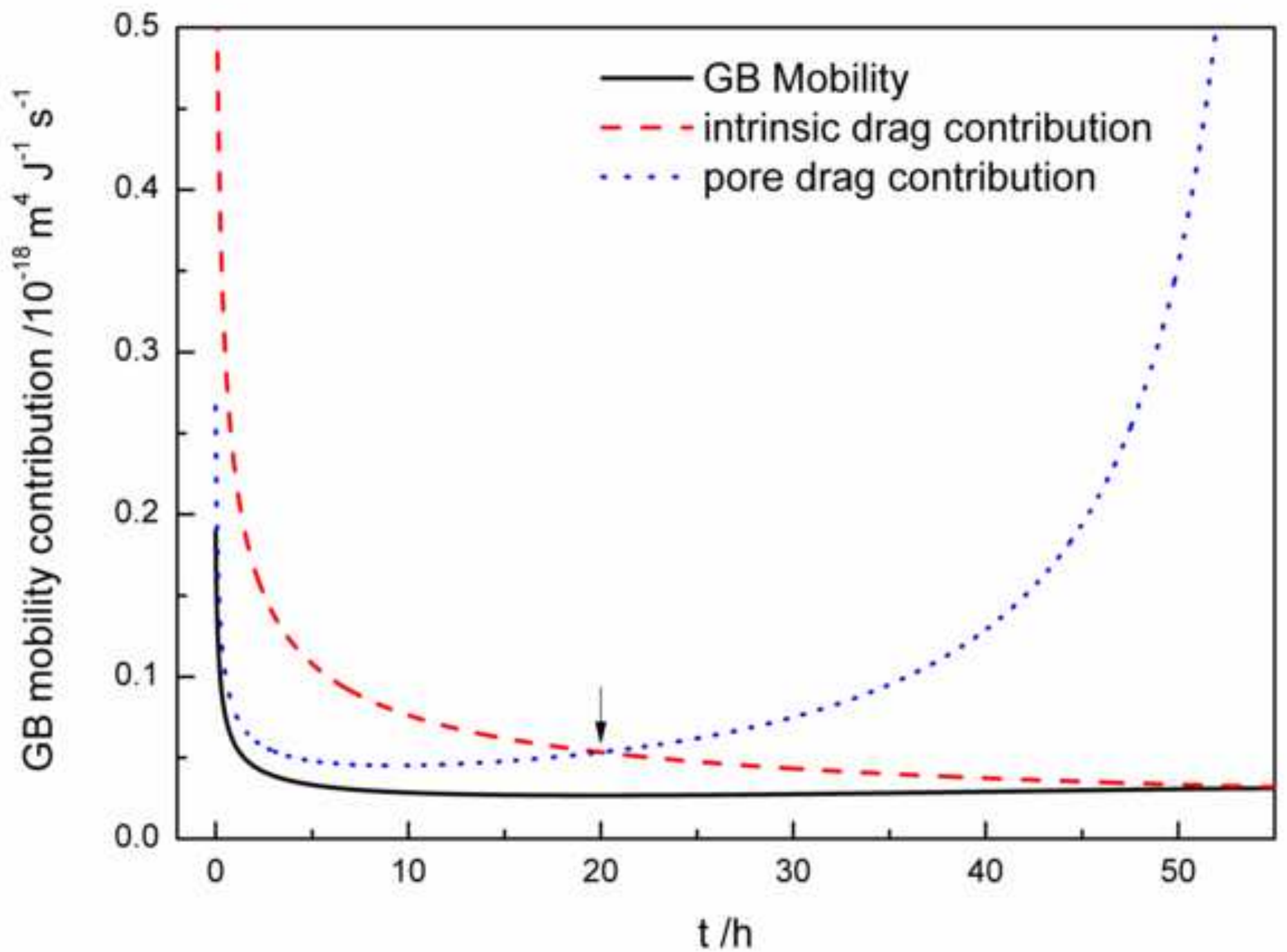


Table 1. Values of parameters used for model calculation.

\begin{tabular}{c|cccc}
\hline Parameter & $m_{g b}$ & $m_{s}$ & $a_{0}(\AA)$ & $\alpha$ \\
\hline Value & 3 & 1 & $5.15[55]$ & 2 \\
\hline Parameter & $\gamma_{g b 0}\left(\mathrm{~J} \mathrm{~m}^{-2}\right)$ & $\gamma_{s 0}\left(\mathrm{~J} \mathrm{~m}^{-2}\right)$ & $\begin{array}{c}\Delta H_{\text {seg }}{ }^{g b} \\
\left(\mathrm{~kJ} \mathrm{~mol}^{-1}\right)\end{array}$ & $\begin{array}{c}\Delta H_{\text {seg }}{ }^{-1} \\
\left(\mathrm{~kJ} \mathrm{~mol}^{-1}\right)\end{array}$ \\
\hline Value & $1.15[24]$ & $1.17[55]$ & $-48.75[55]$ & $-54.80[55]$ \\
\hline
\end{tabular}

